\title{
Vaginal Birth After Caesarean: Any Predictive Model
}

\author{
Ikeanyi Eugene Maduabuchukwu*, Ibrahim Isa Ayuba \\ Department of Obstetrics and Gynecology, Niger Delta University, Amassoma, Nigeria \\ Email address: \\ abuchikeanyi@yahoo.com (I. E. Maduabuchukwu), daddayzee@yahoo.com (I. I. Ayuba) \\ ${ }^{*}$ Corresponding author
}

\section{To cite this article:}

Ikeanyi Eugene Maduabuchukwu, Ibrahim Isa Ayuba. Vaginal Birth After Caesarean: Any Predictive Model. Journal of Gynecology and Obstetrics. Vol. 6, No. 2, 2018, pp. 26-30. doi: 10.11648/j.jgo.20180602.12

Received: March 26, 2018; Accepted: April 15, 2018; Published: May 17, 2018

\begin{abstract}
Background: There is an increased aversion to caesarean birth and high premium to vaginal birth especially in developing countries. Trial of vaginal birth after caesarean is therefore readily accepted and successful vaginal birth highly celebrated especially in sub Saharan Africa. This reduces both the caesarean section rate and repeat caesarean morbidities yet optimal conduct of trial of vaginal birth after caesarean remains a compelling obstetric challenge. This study therefore appraised the correlates of successful trial of vaginal birth after caesarean to contribute data to the increasing evidence for optimal trial of vaginal birth after caesarean. Methods: This was a case series observational study of 334 women who had trial of vaginal birth after a primary caesarean section at a mission hospital between June 2009 and April 2016. Results: Successful VBAC rate in this study was $53.3 \%$. The predictors from the primary CS were non-recurrent indications (OR 2.0, P: 0.01 ), primary CS at tertiary health facility (OR 2.1, P: 0.045) and obstetrician surgeon (OR 1.8, P: 0.02). Others were previous vaginal delivery (OR.3.2, P <0.001), previous VBAC (OR 2.6, P: 0.02), spontaneous labor (OR 1.6, P: 0.06) and induced labor (OR. 0.5, P: 0.01). Conclusion: Trial of vaginal birth after caesarean is a viable option to reduce the increasing caesarean section rate and its associated morbidity especially in high parity settings. It only requires quality management particularly strict selection criteria and conscious labor supervision to optimize its benefits.
\end{abstract}

Keywords: After Caesarean, Predictive, Trial, Vaginal Birth

\section{Introduction}

The incidence of caesarean delivery is on the increase worldwide. Previously during the era of classical uterine incision of early twentieth century, the practice was guided by Craigin's dictum 'once caesarean always caesarean' by 1916. [1] This was informed by high rates and almost certainty of uterine rupture and maternal death due to weak classical upper uterine segment vertical scar prevalent at that time. The introduction of Munro Kerr transverse lower uterine segment (incision) CS (LUSCS) of 1926 [2-3] coinciding with improved surgical skill, anesthesia, blood banking and neonatology services brought about the radical change in the practice with the introduction of trial of vaginal birth after caesarean (TVBAC) with encouraging success in the contemporary obstetrics practice.

As the number of women who have caesarean birth continues to increase [4] and no authentic predictors of successful TVBAC the obstetric complications remain little changed. There are no models that accurately predict women who are likely to have successful TVBAC or to rupture their uteri. [4] There is accruing evidence of serious obstetric complications from multiple caesareans. [4] Non-clinical factors like facility of primary CS, sociodemographic characteristics such as maternal age, socioeconomic status, race, and past obstetric factors have been noted to influence the eligibility for TVBAC. [4] This study was therefore undertaken to evaluate the factors that determine the outcome of TVBAC. The data was hoped to assist in decision making in management of delivery after a prior caesarean.

\section{Materials and Methods}

This was a case series descriptive study that took place at St Philomena Catholic Hospital (SPCH) Benin City Edo state, Nigeria between June 2009 and April 2016. SPCH is a leading long serving referral mission hospital strategically located at the city. Edo state is one of the oil rich states in 
Nigeria with the residents predominantly government workers and traders. The records of the 407 deliveries after a previous caesarean delivery within the reviewed period were retrieved and reviewed. The sources of information were labor ward and labor ward theatre records and patients case notes. Information on the maternal age, parity, booking status, and mode of delivery were extracted. The main outcome measures were rate of successful vagina birth after caesarean (VBAC) and the repeat caesarean section rate.

At the first prenatal visit each subject was properly evaluated with emphasis on past obstetric history; indication for the primary caesarean birth, date of the surgery from which the inter-pregnancy interval was calculated, complications thereof especially wound complications, the facility and cadre of surgeon of primary caesarean delivery. Clinical palpation and ultrasound scan were used for diagnosis. Clinical and or radiological pelvimetry examination was done for each parturient to assess for pelvic adequacy especially those who had no previous vaginal delivery. Those subjects with interpregnancy interval of more than 6 months; who fulfilled all conditions for vaginal delivery, including a prior caesarean section in a setting of high level care, satisfactory post-operative wound condition, well-motivated and consented to TVBAC were allowed to attempt TVBAC. These were included in the study. Nonrecurrent indications for the primary caesarean section for the purpose of this study were previous fetal distress, placenta praevia or abruption, abnormal lie, malpresentation, gestational hypertension, and primary CS for elderly nulliparity. The recurrent indications were obstructed labor, cephalopelvic disproportion (CPD) and confirmed contracted pelvis. Each parturient readily gave consent for TVBAC. Two units of blood were typed and cross matched and made available in the blood bank, the anesthetist, neonatologist and theatre staff were put at alert when the subjects were in labor. Cardio tocogram and partogram were used to monitor the labor process. Elective repeat caesarean section was offered on obstetric indications; placenta praevia, multiple gestation, breech presentation, cord presentation or prolapse, excessive fetal weight, those who opted for a repeat caesarean birth that declined consent for TVBAC and interpregnancy interval shorter than six months. These were so excluded from the analysis. The study was approved by hospital Research and Ethics Committee (REC). The process of data collection did not involve subject identifiers therefore did not require individual subject consent

Statistical analysis was done using EPI INFO Version 3.5.1 and INSTAT statistical packages. Fisher's exact test was done where applicable with statistical significance based on $95 \%$ confidence interval excluding nullity of one and two sided $\mathrm{P}<.05$.

\section{Results}

A total of 407 women had their next delivery after a primary caesarean delivery in this center in the reviewed period. Three hundred and thirty four $(82.1 \%)$ of them fulfilled the selection criteria for trial of vaginal birth after caesarean (TVBAC) and subsequent analyses were on them. The rest had planned caesarean delivery for various obstetric indications.

Table 1 revealed that $178(53.3 \%)$ of the women had successful vaginal birth after a previous caesarean section (VBAC) and $156(46.7 \%)$ had emergency repeat caesarean birth due to failed trial of vaginal birth after caesarean. The women were aged $22-45$ years with $137(41.0 \%$ ) within $30-34$ years age bracket. The age of the women did not significantly influence their mode of delivery. The women who had their second delivery following caesarean delivery in their first pregnancy were significantly more than half at reduced odds of successful vaginal delivery (OR $0.3, \mathrm{P}<.001)$. The multiparous women on the other hand were more than thrice at increased likelihood of successful vaginal birth after a previous caesarean section (OR 3.3, $\mathrm{P}<.001$ ). This finding was statistically significant. The booking status appeared not to have influence on the labor outcome of the women $(\mathrm{P}=1.00)$.

Table 1. Sociodemographic Profile of Mothers and Trial of Vaginal Birth after Caesarean $\{N=334)$.

\begin{tabular}{|c|c|c|c|c|}
\hline Characteristic/Variables & VBAC $n=178$ & EMCS $n=156$ & OR $(95 \%$ CI $)$ & $P$ value \\
\hline \multicolumn{5}{|l|}{ Age } \\
\hline $20-24$ & 6 & 9 & 0.57 & 0.31 \\
\hline $30-34$ & 69 & 68 & 0.82 & 0.38 \\
\hline$\geq 35$ & 38 & 26 & 1.36 & 0.33 \\
\hline Total & $178(53.3)$ & $156(46.7)$ & & \\
\hline 1 & 84 & 116 & $0.31(0.19,0.49)$ & $<0.001$ \\
\hline$\geq 2$ & 94 & 40 & $3.25(2.04,5.16)$ & $<0.001$ \\
\hline \multicolumn{5}{|l|}{ Booking status } \\
\hline Booked & 172 & 151 & 0.95 & 1.00 \\
\hline Unbooked & 6 & 5 & & \\
\hline
\end{tabular}

From Table 2, the women who had their previous caesarean section for recurrent indications were about half at reduced likelihood of successful trial of vaginal birth after caesarean (TVBAC) $(\mathrm{OR} 0.51, \mathrm{P}=.005)$ while non-recurrent indications resulted in almost twice increased vaginal birth rate (OR1.95, $\mathrm{P}=.005)$. The observed differences were 
statistically significant. Analysis of the cadre of the surgeons in the first caesarean delivery showed that the women operated by the medical officers were at more than $90 \%$ reduced likelihood of having successful vaginal delivery (OR $0.07, \mathrm{P}<.001)$. The observed difference was statistically significant. There was about $60 \%$ increased likelihood of successful vaginal delivery among the women who had their primary caesarean delivery from trainee obstetricians (resident doctors) although this was not statistically significant (OR 1.55, $\mathrm{P}=0.18$ ). This increased to a significant $80 \%$ among those who had their caesarean delivery from obstetricians $(\mathrm{OR}=1.75, \mathrm{P}=.024)$. The women who had their primary $\mathrm{CS}$ in the private health facilities were more than half at reduced likelihood of successful TVBAC (OR 0.41, $\mathrm{P}=.012$ ). This was statistically significant. On the other hand, women who had their caesarean delivery in the Teaching (tertiary) hospitals were statistically significant more than twice at increased likelihood of successful TVBAC (OR 2.1, $\mathrm{P}=.045$ ). Approximately 6 out of every 10 of $68 \%$ (227) majority of the women who had spontaneous labor and about 2 out of every 10 of $12 \%$ who had augmentation of labor had successful VBAC None of these observations was statistically significant. Half of about $20 \%$ who had induction of labor had failed TVBAC and this was significant. This meant that while there was about $60 \%$ increased chance of successful VBAC among the women who went into spontaneous labor, there was less than $15 \%$ increased chance of successful augmentation of labor and a significant half(OR $0.50, \mathrm{P}=.013)$ reduced chance of successful induction of labor (Table 2) in TVBAC. Women who have experienced previous vaginal delivery had thrice increased likelihood of having successful TVBAC (OR 3.17, $\mathrm{P}<.0001)$ while there was about $70 \%$ reduced chance in those who have never had successful vaginal delivery. Both observations were statistically significant. A further subgroup analysis of women who had previous vaginal delivery showed that those women who had their vaginal delivery prior to caesarean delivery had significant reduction in chance of successful TVBAC $(\mathrm{OR}=0.38, \mathrm{P}=.022)$ when compared with those who had previous VBAC with about tripled likelihood of another successful vaginal delivery (OR 2.64, $\mathrm{P}=.022$ ). This was statistically significant. Those who had at least a previous vaginal delivery before and after a previous caesarean birth had more than twice (OR 2.16, $\mathrm{P}=0.40$ ) increased chance of a successful repeat VBAC. This was not statistically significant.

Table 2. Putative Factors influencing the Outcome of Trial of Vaginal Birth after Caesarean (N=334).

\begin{tabular}{|c|c|c|c|c|}
\hline Factor/Variables & VBAC $n=178$ & Repeat CS n=156 & OR(95\% CI) & P-value \\
\hline \multicolumn{5}{|c|}{ Indication for primary CS } \\
\hline Recurrent & 45 & 62 & $0.51(0.32,0.82)$ & 0.005 \\
\hline Non-recurrent & 133 & 94 & $1.95(1.22,3.11)$ & \\
\hline \multicolumn{5}{|c|}{ Level Surgeon Primary CS } \\
\hline Medical officer & 3 & 30 & $0.07(0.021,0.24)$ & $<0.001$ \\
\hline GP Consultant & 3 & 2 & 1.32 & 1.00 \\
\hline O \&G Resident & 33 & 20 & 1.55 & 0.18 \\
\hline Obstetrician & 133 & 98 & $1.75(1.10,2.80)$ & 0.024 \\
\hline Others & 6 & 6 & 0.87 & 1.00 \\
\hline \multicolumn{5}{|l|}{ Facility primary CS } \\
\hline Teaching hosp. & 28 & 13 & $2.1(1.02,4.12)$ & 0.045 \\
\hline Mission hosp. & 125 & 107 & 1.08 & 0.81 \\
\hline Private hosp. & 14 & 27 & $0.41(0.21,0.81)$ & 0.012 \\
\hline Specialist hosp. & 11 & 9 & 1.08 & 1.00 \\
\hline \multicolumn{5}{|c|}{ Previous vaginal delivery } \\
\hline Yes & 94 & 40 & $3.17(2.0,5.05)$ & $<0.001$ \\
\hline No & 84 & 116 & $0.32(0.20,0.5)$ & \\
\hline \multicolumn{5}{|c|}{ Sequence prev. vaginal delivery } \\
\hline Before primary CS & 47 & 29 & $0.38(0.17,0.85)$ & 0.022 \\
\hline After primary CS & 47 & 11 & $2.64(1.18,5.89)$ & \\
\hline \multicolumn{5}{|l|}{ Type of labor } \\
\hline Spontaneous & 129 & 98 & $1.56(0.98,2.47)$ & 0.061 \\
\hline Induced & 26 & 40 & $0.50(0.29,0.86)$ & 0.013 \\
\hline Augmented & 23 & 18 & 1.14 & 0.74 \\
\hline
\end{tabular}

There was $10-30 \%$ increase likelihood of successful TVBAC with fetal weight within a range of 3000 grams (OR 1.27, P: 0.31 ) and less than 4000grams (OR 1.09, P: 0.81) respectively (Figure 1). 


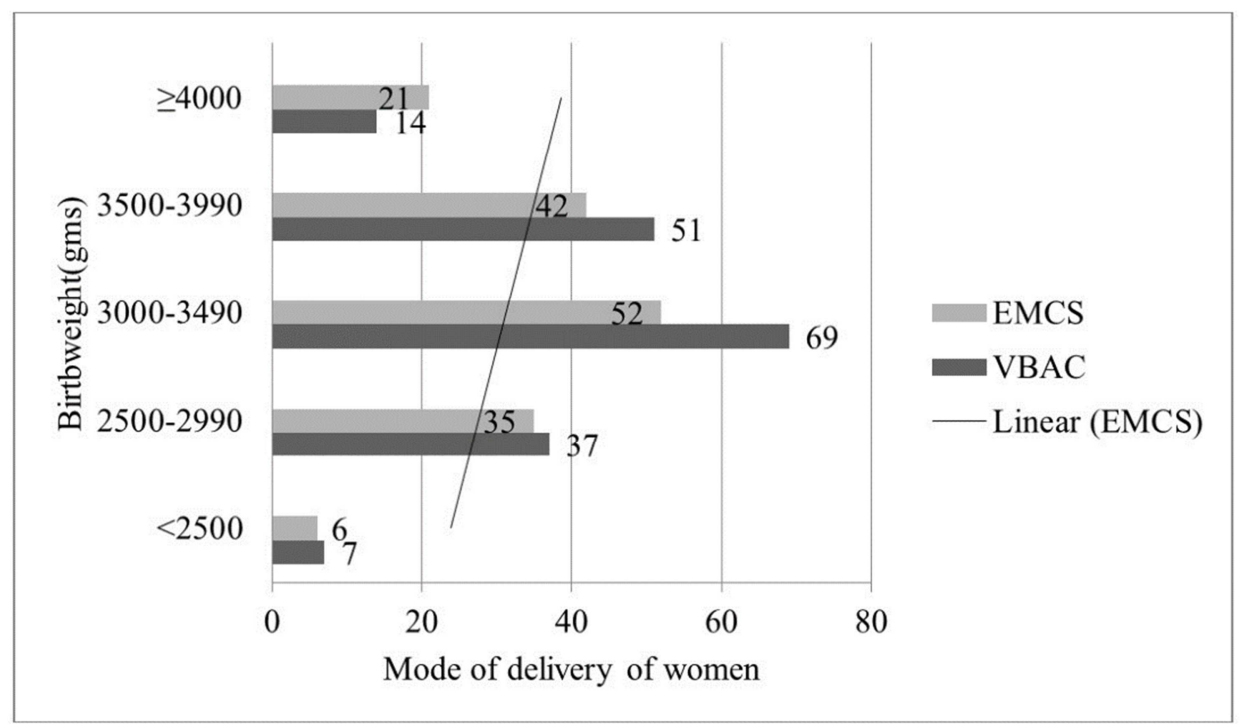

Figure 1. Fetal weight and Trial of vaginal birth after caesarean.

However, this was not statistical significant. Analysis of maternal weight and TVBAC revealed a trend of decrease of rate of successful VBAC from the subset of the women of normal body mass index (BMI) (OR 1.58, P 0.17) at booking to a significant level among the obese subset (OR 0.58, CI: 0.38-0.90, P: 0.016) Figure 2.

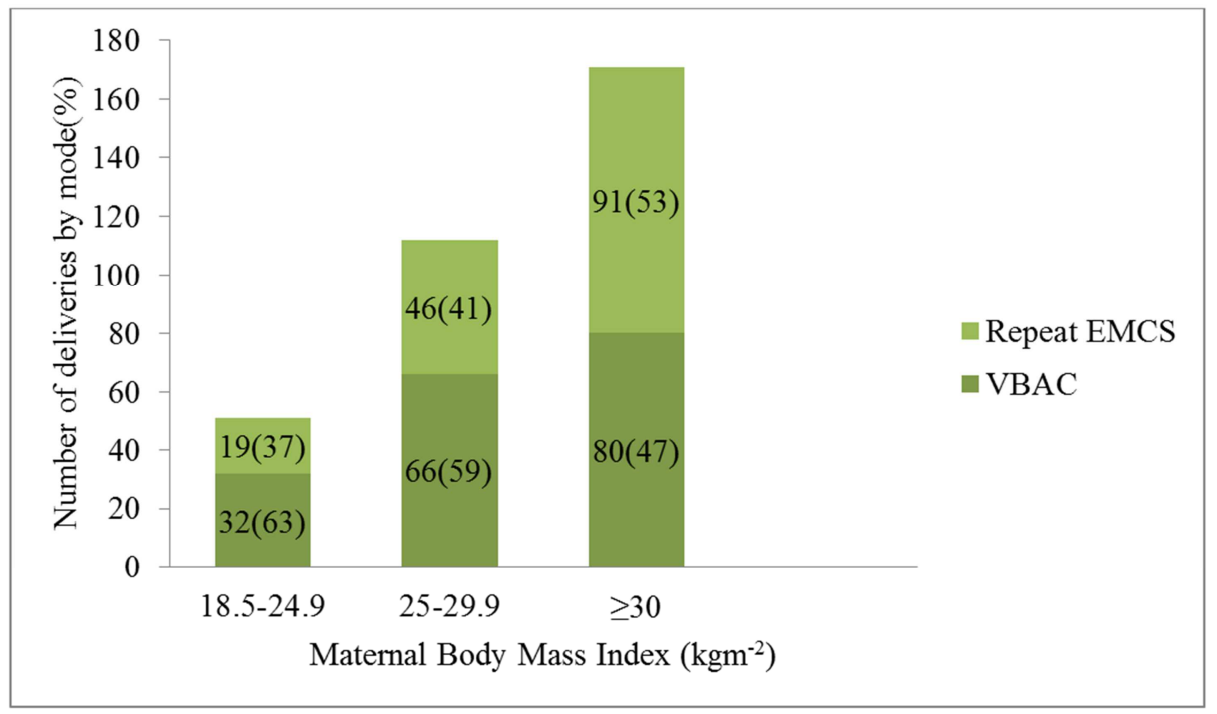

Figure 2. Maternal Body Mass Index and Trial of Vaginal Birth after Caesarean.

\section{Discussion}

Quality trial of vaginal birth after caesarean is an invaluable option for reduction of caesarean section rate and the associated morbidity and mortality. From this study primary CS for non-recurrent indication performed by skilled surgeon in a good level care health facility that ensures good incision placement and scar healing and integrity, previous VBAC, average size fetus in normal weight woman and spontaneous labor were associated with optimal outcome of TVBAC. Skilled caesarean with the proper placement of lower uterine segment incision ensures a scar that withstands normal uterine contractions. About $80 \%$ of the women with a prior caesarean birth were eligible for this study comparable to $28-82 \%$ in previous studies. [4-5] The rate of successful VBAC in this study was $53.3 \%$ comparable to $49-87 \%$ reported by other researchers. [4-6] Non -recurrent indications for the primary caesarean delivery appeared to predict successful TVBAC. This compared with reports from other authors. [6-9] Women who had previous vaginal birth had increased chances of success in TVBAC consistent to other reports. [5-6] Much as a prior vaginal delivery seemed to favor successful VBAC as in earlier reports $[6,8]$, this data further appeared to suggest that those who had previous VBAC were comparatively at higher likelihood of another successful TVBAC compared with those who had only normal vaginal deliveries before their primary caesarean birth corroborating another report in literature. [10] This data revealed that the likelihood of successful TVBAC was 
comparable in all the women who were undergoing their first TVBAC irrespective of prior vaginal births or not. This may be due to increasing fetal weight with subsequent pregnancies. It is evident that the risk of uterine rupture; the most feared complication of TVBAC, decreases with subsequent vaginal deliveries after the first successful VBAC. [6, 11] Again it was evident that maternal and perinatal morbidity increase if TVBAC fails emphasizing careful selection of cases. [6, 12-13] Maternal weight seemed to influence the success rate of TVBAC. This data appeared to reveal maximal success rate of VBAC among the normal weight mothers and lowest among the obese mothers. The contrast was true for the failure rate and subsequent repeat caesarean delivery in keeping with reports by other authors. [1, 10, 14-15] Similarly, as evidenced in this data, average fetal weight appeared to be associated with the best outcome relative to the extremes of fetal weight.

The conclusions of this study may be limited by its sample size, retrospective and a hospital based data. A wellstructured prospective multicenter and randomized study will ensure more authentic conclusions. This however, may provoke some ethical concerns.

\section{Conclusion}

Trial of Vaginal birth after caesarean is an invaluable option especially in high parity settings in this era of increasing caesarean section rates. This data emphasized the crucial need for conscious and strict selection criteria and quality labor supervision to optimize TVBAC.

\section{Recommendations}

Trial of vaginal birth after caesarean should be conducted for parturient with a previous caesarean section for nonrecurrent cause performed by a skilled surgeon in a high level care health facility best if labor is spontaneous in a woman with a previous VBAC. The contrary is true in primary CS for a recurrent cause performed by a medical officer in a low level care private hospital and induced labor in a woman without a previous VBAC irrespective of the number of her previous vaginal delivery before the primary caesarean.

\section{References}

[1] Cragin E. Conservation in Obstetrics. N Y Med J 1961; 140:1.
[2] Cunningham GF, Leveno KJ, Bloom SL Hauth JC, Rouse DJ, Spong CY. Prior caesarian delivery. In: F Cunningham GF, editor. Williams Obstetrics. 23rd ed. New York: McGraw Hill; 2010. pp. 565-76.

[3] John L. Powell. The Kerr Incision: John Martin Munro Kerr 1868-1960). Journal of Pelvic Surgery 2001; 7(3): 177-178.

[4] Guise JM, Eden K, Emeis C, Denman MA, Marshall N, Fu RR et al. Vaginal birth after caesarean: new insights. Evid Rep Technol Assess 2010; (191): 1-397.

[5] Pratiksha Gupta, Ishrat Jahan, Gelabhai R. Jograjiya. Is vaginal delivery safe after previous lower segment caesarean section in developing country. Nig Med. J 2014; 55(3):260265 .

[6] Guidelines for Vaginal birth After Previous Caesarea Birth: Society of Obstetrics and Gynaecology of Canada (SOGC) clinical Practice Guidelines. J Obstet Gynaecol Can 2005; 27(2): 164-174.

[7] Weinstein D, Benshushan A, Tanos V, Zilberstein R, Rojansky N. Predictive score for Vaginal birth after caesarean section. Am J Obstet Gynecol 1996; 174:192-8

[8] Flamm BL, Geiger AM. Vaginal birth after Caesarean delivery: an admission scoring system. Obstet Gynecol 1997; 90:907-10.

[9] Olagbuji B, Ezeanochie M, Okonofua F. Predictors of successful vaginal delivery after previous caesarean section in a Nigerian tertiary hospital. J Obstet Gynaecol 2010; 30(6): $582-5$.

[10] Aaron B Caughey, Carl V Smith. Vaginal Birth after Caesarean. Medscape 2015.

[11] Shimonovitz S, Botosneano A, Hochner-Celnikier D. Successful first vaginal birth after Cesarean section: a predictor of reduced risk for uterine rupture in subsequent deliveries. Indian Med Assoc J 2000; 2:526-8.

[12] Biswass A. Management of previous caesarean section. Curr Opin Obstet Gynecol 2003; 15: 123-9.

[13] Eugene M Ikeanyi, Alphonsus N Onyiriuka. Comparative Analysis of Maternal and Perinatal Outcomes of Trial of Vaginal Birth and Planned Repeat Caesarean section following a prior Caesarean BJMMR 2016;14(1):1-9.

[14] Durnwald C, Ehrenberg H, Mercer H. The impact of maternal obesity and weight gain on VBAC success. Am J Obstet Gynecol 2003; 189:S205.

[15] Carroll CS Sr,. Magann EF, Chauhaan SP, Klauser CK, Morrison JC. Vaginal birth after caesarean section versus elective repeat caesarean delivery: weight-based outcomes. Am J Obstet Gynecol 2003, 188: 1516-1520. 